\title{
Death begets failure in the heart
}

\author{
Roger S.-Y. Foo, ${ }^{1}$ Kartik Mani, ${ }^{1}$ and Richard N. Kitsis ${ }^{1,2}$
}

${ }^{1}$ Departments of Medicine and Cell Biology, Cardiovascular Research Center, and ${ }^{2}$ Cancer Center, Albert Einstein College of Medicine, Bronx, New York, USA.

\begin{abstract}
Recently, low - but abnormal - rates of cardiomyocyte apoptosis have been observed in failing human hearts. Genetic and pharmacological studies suggest that this cell death is causally linked to heart failure in rodent models. Herein, we review these data and discuss potential therapeutic implications.
\end{abstract}

\section{Introduction}

Heart failure is a heterogeneous syndrome that can result from primary cardiomyopathies or, more commonly, myocardial infarction, hypertension, and valvular heart disease, among other disorders. The prevalence of heart failure has increased dramatically as modern therapies have reduced the mortality of acute myocardial infarction. However, current treatments for heart failure are woefully inadequate, and the availability of hearts for transplantation is severely limited. Even those therapies that successfully target biologically relevant pathways (e.g., $\beta$-adrenergic receptor blockers, angiotensin-converting enzyme inhibitors) become less effective with time (1). These limitations underscore the need for understanding the biology of heart failure at the most fundamental level.

The underlying cause of heart failure has remained an enigma since this syndrome was first described by Richard Lower in 1669 (2). Multiple mechanisms have been proposed, including desensitization of $\beta$-adrenergic receptor signaling (3), dysregulation of excitation-contraction coupling $(4,5)$, alterations in cytoskeletal proteins (6), myosin isoform switches (7), and dysfunctional energy utilization $(8,9)$, all topics covered elsewhere in this series. Indeed, these mechanisms have been implicated in the progressive loss of contractile function in heart failure. On the other hand, there is a longstanding notion that heart failure involves not only myocyte contractile dysfunction, but also cell "drop-out." The loss of myocytes would be predicted to decrease contractility and promote slippage of muscle bundles, wall thinning, and dilatation - the archetypical changes observed in heart failure.

In this review, we first discuss the evidence that cardiomyocyte death plays a mechanistic role in heart failure and go on to consider how this mechanism may provide a target for novel therapies.

\section{Central apoptosis pathways}

Cell death can occur by apoptosis, necrosis, or perhaps autophagy. A framework has been described for apoptosis, a highly regulated cell suicide process that is hard wired into all metazoan cells (10). Much less is known about necrosis, although recent work suggests that this form of cell death may be actively regulated and is not necessarily accidental $(11,12)$. Autophagy is an important recycling process in which macromolecules are degraded in the lysosome so that their components can be used as energy sub-

Nonstandard abbreviations used: ARC, apoptosis repressor with a CARD (caspase recruitment domain); $\mathrm{BH} 3$, Bcl-2 homology domain 3; DISC, death-inducing signaling complex; FADD, Fas-associated via death domain; Gaq, $\alpha$ subunit of Gq; IP3R, inositol 1,4,5-triphosphate receptor; XIAP, X-linked inhibitor of apoptosis.

Conflict of interest: The authors have declared that no conflict of interest exists.

Citation for this article: J. Clin. Invest. 115:565-571 (2005).

doi:10.1172/JCI200524569. strates by the cell (13). Whether autophagy functions as a death process remains controversial $(11,14)$.

Apoptosis is mediated by 2 evolutionarily conserved central death pathways: the extrinsic pathway, which utilizes cell surface death receptors; and the intrinsic pathway, involving mitochondria and the ER (Figure 1) (10). In the extrinsic pathway, death ligands (e.g., FasL) initiate apoptosis by binding their cognate receptors (15). This stimulates the recruitment of the adaptor protein Fas-associated via death domain (FADD), which then recruits procaspase- 8 into the death-inducing signaling complex (DISC) $(16-18)$. Procaspase- 8 is activated by dimerization in this complex and subsequently cleaves and activates procaspase- 3 and other downstream procaspases (19).

In contrast to the extrinsic pathway that mediates a specialized subset of death signals, the intrinsic pathway transduces a wide variety of extracellular and intracellular stimuli including loss of survival/trophic factors, toxins, radiation, hypoxia, oxidative stress, ischemia-reperfusion, and DNA damage. Although a myriad of peripheral pathways connect these signals with the central death machinery, each ultimately feeds into a variety of proapoptotic Bcl-2 proteins that possess only Bcl-2 homology domain 3 (BH3-only proteins) and the proapoptotic multidomain Bcl-2 proteins Bax and Bak (20). These proteins undergo activation through a diversity of mechanisms to trigger the release of mitochondrial apoptogens, such as cytochrome $c$, into the cytoplasm (21-27). Once in the cytoplasm, cytochrome $c$ binds Apaf- 1 along with dATP. This stimulates Apaf-1 to homo-oligomerize and recruit procaspase- 9 into the multiprotein complex called the apoptosome (28-39). Within the apoptosome, procaspase- 9 is activated by dimerization, after which it cleaves and activates downstream procaspases. Bid, a $\mathrm{BH} 3$-only protein, unites the extrinsic and intrinsic pathways: following cleavage by caspase-8, Bid's C-terminal portion translocates to the mitochondria and triggers apoptogen release (40-42).

The extrinsic and intrinsic pathways are held in check by a variety of endogenous inhibitors of apoptosis. FLICE-like (Fas-associated death domain protein-like-interleukin-1-converting enzyme-like) inhibitory protein (FLIP), whose expression is highly enriched in striated muscle, binds to and inhibits procaspase- 8 in the DISC (43). Antiapoptotic Bcl-2 proteins, such as Bcl-2 and Bcl- $\mathrm{x}_{\mathrm{L}}$, inhibit mitochondrial apoptogen release through biochemical mechanisms that are still incompletely understood (10). Ku-70 and humanin bind Bax and block its conformational activation and translocation to the mitochondria $(44,45)$. X-linked inhibitor of apoptosis (XIAP) and related proteins that contain baculovirus inhibitor of apoptosis repeats bind to and inhibit already activated caspases-9, -3 , and -7 , as well as interfering with procaspase- 9 dimerization and activation (46-50). Each of these inhibitors act on circumscribed portions of either the extrinsic or intrinsic pathway. In contrast, 


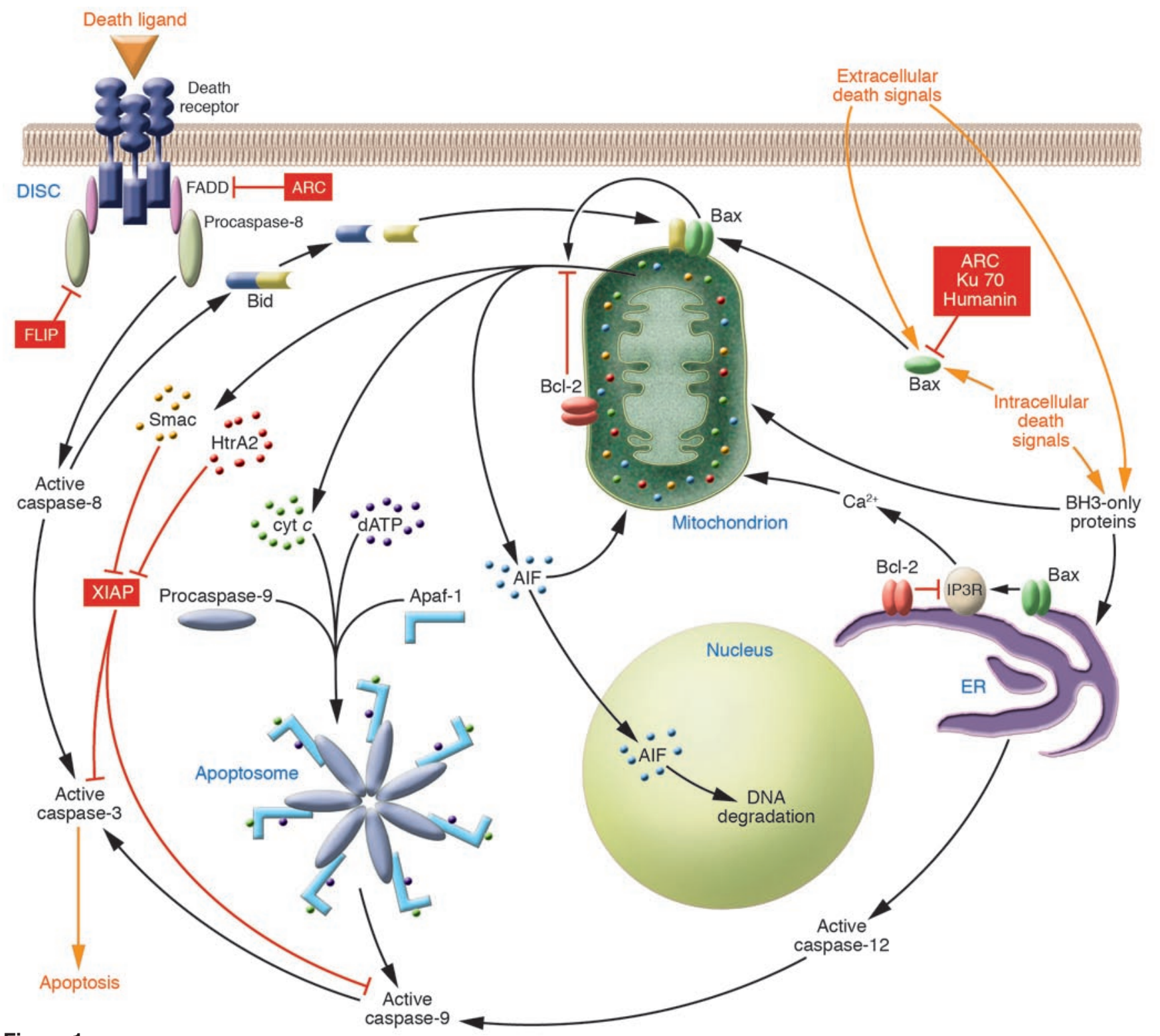

Figure 1

In the extrinsic pathway, ligand binding induces death receptors to recruit FADD, which recruits procaspase-8. Within this complex (DISC), procaspase-8 dimerizes and activates. Caspase-8 proteolytically activates procaspase-3, which cleaves cellular proteins causing cell death. In the intrinsic pathway, extracellular and intracellular stimuli signal to the mitochondria through a variety of BH3-only proteins (e.g., Bid) and through Bax, which translocates to and inserts into the outer mitochondrial membrane. Bax and Bak (not shown) stimulate the release of cytochrome $c$ (cyt $c$ ) and other apoptogens. Once released, cytochrome $c$ binds Apaf- 1 along with dATP. This triggers oligomerization of Apaf-1 and recruitment of procaspase- 9 . Within this complex (apoptosome), procaspase-9 dimerizes and activates. Caspase-9 proteolytically activates procaspase-3. Caspase-8 also cleaves Bid, and its C-terminal fragment translocates to the mitochondria, where it activates Bax and Bak stimulating apoptogen release. FLIP binds and inhibits procaspase-8 in the DISC. ARC binds Fas, FADD, and procaspase-8, inhibiting DISC assembly. ARC, Ku-70, and Humanin bind Bax and inhibit its conformational activation and translocation. Bcl-2 and Bcl- $\mathrm{x}_{\mathrm{L}}$ (not shown) inhibit mitochondrial apoptogen release. XIAP binds and inhibits activated caspase-9 and -3. Once cytoplasmic, the mitochondrial apoptogens Smac and HtrA2 bind XIAP, displacing and disinhibiting caspases. HtrA2 also has a serine protease activity that cleaves XIAP (not shown). AIF translocates to the nucleus and, in conjunction with a presumed endonuclease, mediates large-scale DNA fragmentation. A subset of intrinsic pathway death signals stimulate the ER, possibly through BH3-only proteins. This causes the release of intraluminal $\mathrm{Ca}^{2+}$ into the cytoplasm, which is mediated by Bax and Bcl-2 through the IP3R. Ca ${ }^{2+}$ translocates to the mitochondrial matrix and stimulates opening of the mitochondrial permeability transition pore in the inner membrane (not shown), which indirectly results in apoptogen release. The ER pathway may also activate procaspase-12, which can cleave and activate procaspase-9 independently of apoptosome formation (see text).

apoptosis repressor with a CARD (ARC), which is expressed preferentially in striated muscle and some neurons, antagonizes both central apoptosis pathways (51). The extrinsic pathway is inhibited by ARC's direct interactions with Fas, FADD, and procaspase-8, which prevent DISC assembly, while the intrinsic pathway is inhibited by direct binding and inhibition of Bax (51-53).

Efficient cell killing usually requires neutralization of inhibitory pathways as well as activation of effector mechanisms. This 
is best illustrated in mammalian cells by the ability of sympathetic neurons to withstand the usual toxic effects of direct intracellular injection of cytochrome $c$, a resistance attributable to XIAP (54). In most cell types, XIAP's tonic inhibition of caspases is relieved by the release of the mitochondrial apoptogens Smac/DIABLO and Omi/HtrA2, which directly bind XIAP, thereby displacing caspases (23-25). In addition, Omi/HtrA2 has a serine protease activity that cleaves XIAP, resulting in its irreversible inhibition (55).

Recently, the endoplasmic reticulum has been recognized as an important organelle in the intrinsic pathway. In addition to its role in cellular responses to traditional ER stresses, such as misfolded proteins, this organelle appears to be critical in mediating cell death elicited by a subset of stimuli originating outside of the ER, such as oxidative stress (56). Similar to their roles in transducing upstream signals to the mitochondria, $\mathrm{BH} 3$-only proteins appear to relay upstream death signals to the ER (57). The death signal output from the ER can take several forms. First, certain death stimuli increase cytoplasmic $\mathrm{Ca}^{2+}$ concentrations, a process controlled by $\mathrm{Bax}$ and $\mathrm{Bcl}-2$ in the ER membrane through interactions between $\mathrm{Bcl}-2$ and the inositol 1,4,5-trisphosphate receptor (IP3R), an ER $\mathrm{Ca}^{2+}$ release channel $(56,58)$. Increased cytoplasmic $\mathrm{Ca}^{2+}$ can result in $\mathrm{Ca}^{2+}$ overloading of the mitochondrial matrix and opening of the mitochondrial permeability transition pore. This, in turn, can lead to permeabilization and depolarization of the inner mitochondrial membrane, gross mitochondrial swelling, rupture of the outer mitochondrial membrane, and apoptogen release (59). The relevance of this mechanism may be dependent on cell context and stimulus, however, as apoptogen release in some instances of apoptosis is limited to much more subtle features of mitochondrial remodeling (60). A second ER death output is activation of procaspase-12 (61). Procaspase-12 can be activated through the intrinsic ER machinery (62) or cleavage by calpain, which can, in turn, be activated by elevations in cytoplasmic $\mathrm{Ca}^{2+}$ (63). Once activated, caspase- 12 can translocate to the cytoplasm and cleave and activate procaspase- 9 independently of apoptosome formation (64). This mechanism may provide a means by which the ER can carry out the distal steps in the intrinsic pathway independently of mitochondria. Although knockout mice have uncovered an essential role for procaspase-12 in ER stress-induced apoptosis (61), the applicability of this mechanism to humans is less clear due to procaspase-12 polymorphisms in some populations that encode nonsense mutations (65).

\section{Lessons from myocardial ischemia-reperfusion}

The significance of these apoptotic pathways in cardiomyocytes is most clearly revealed in the context of ischemia-reperfusion, a model characterized by a robust burst of apoptosis over a limited time frame $(66,67)$. Ischemia-reperfusion activates both the extrinsic and intrinsic pathways. Moreover, a variety of genetic studies indicate that both pathways play critical roles in the genesis of ischemia-reperfusion-induced myocardial infarction. Thus, a loss of function mutation in the death receptor Fas (lpr mouse) results in a 64\% decrease in cardiomyocyte apoptosis and a $63 \%$ decrease in infarct size following ischemia-reperfusion in vivo (68). The source of death ligands during ischemia-reperfusion may be the heart cells (myocytes or nonmyocytes) themselves, as transudates from isolated perfused wild-type hearts contain FasL, TNF- $\alpha$, and TNF-related apoptosis-inducing ligand (TRAIL) in the reperfusion phase (69). Similarly, infarct size following ischemia-reperfusion is reduced by $53 \%$ in mice lacking $\mathrm{Bid}$, and this is accompanied by reduced cardiac dysfunction (70). These data implicate the extrinsic pathway in cardiomyocyte apop- tosis and suggest that the Bid connection between the extrinsic and intrinsic pathways is important for efficient killing in this model. Additional genetic data underscore the importance of the intrinsic pathway in cardiomyocyte apoptosis following ischemia-reperfusion. Myocardial overexpression of $\mathrm{Bcl}-2$, an antiapoptotic protein that inhibits cytochrome $c$ release, reduces infarct size by $48-64 \%$ (71, 72). Likewise, cardiac-specific expression of either of 2 independent procaspase- 9 dominant negative alleles decreases infarct size by $53 \%$ and $68 \%$, with amelioration of cardiac functional abnormalities (70). These data provide proof of concept that cardiomyocyte apoptosis is a causal component of ischemia-reperfusion injury.

\section{Does myocyte apoptosis occur during heart failure?}

In contrast to ischemia-reperfusion, heart failure is characterized by very low - but abnormal - levels of cardiomyocyte death that persist for months to years. Questions have been raised as to whether myocyte loss in heart failure occurs primarily by apoptosis $(73,74)$. Thus far, however, this issue is unresolved, as only indirect markers have been used to characterize types of cell death. The most rigorous data demonstrate apoptosis rates of $0.08-0.25 \%$ in patients with end-stage dilated cardiomyopathy compared with $0.001-0.002 \%$ in controls $(73,75,76)$. But is it reasonable to believe that rates this low could have a detectable impact on the pathogenesis of heart failure?

\section{Does cardiomyocyte apoptosis play a causal role in heart failure?}

This issue was addressed directly using transgenic mice with heartrestricted expression of a procaspase-8 fusion protein, whose dimerization and activation could be induced by administration of a drug (Figure 2) (77). Not surprisingly, within hours of acutely activating caspase-8, these transgenic mice died due to extensive cardiac damage. Interestingly, however, transgenic mice that never received the dimerizing drug died spontaneously over 2-6 months of a profound dilated cardiomyopathy. In contrast, longevity and cardiac function were normal in mice that expressed lower levels of the transgene protein and mice that expressed similar levels of an identical transgene protein except for a point mutation in the catalytic cysteine of the caspase. The explanation for the cardiomyopathy in the highexpressing inducible caspase-8-transgenic mice proved to be low rates of cardiomyocyte apoptosis: $0.023 \%$ as compared with $0.002 \%$ in controls. These data demonstrate that very low, albeit abnormal, rates of cardiomyocyte apoptosis are sufficient to cause lethal dilated cardiomyopathy. Given that the rates of cardiomyocyte apoptosis in patients with end-stage dilated cardiomyopathy are 5- to 10-fold higher than those in this transgenic model $(73,75,76)$, myocyte apoptosis may also play a causal role in human heart failure. To test whether apoptosis is required for the development of cardiomyopathy in this model, a broad-spectrum caspase inhibitor was administered systemically starting before cardiac decompensation. Caspase inhibition abrogated cardiac dilatation and markedly ameliorated contractile dysfunction. These experiments provide direct evidence that low levels of cardiomyocyte apoptosis may be a causal component of heart failure.

Support for the importance of cardiomyocyte apoptosis in heart failure is also provided by another, more physiological model (Figure 2). Gq transduces humoral (e.g., angiotensin II) and mechanical stimuli that are important in cardiac hypertrophy (78). Myocardial overexpression of the $\alpha$ subunit of Gq (Goq) bypasses the need for stimulus and elicits cardiac hypertrophy and dilated cardiomyopathy (79-81). This phenotype is accompanied by cardiomyocyte 


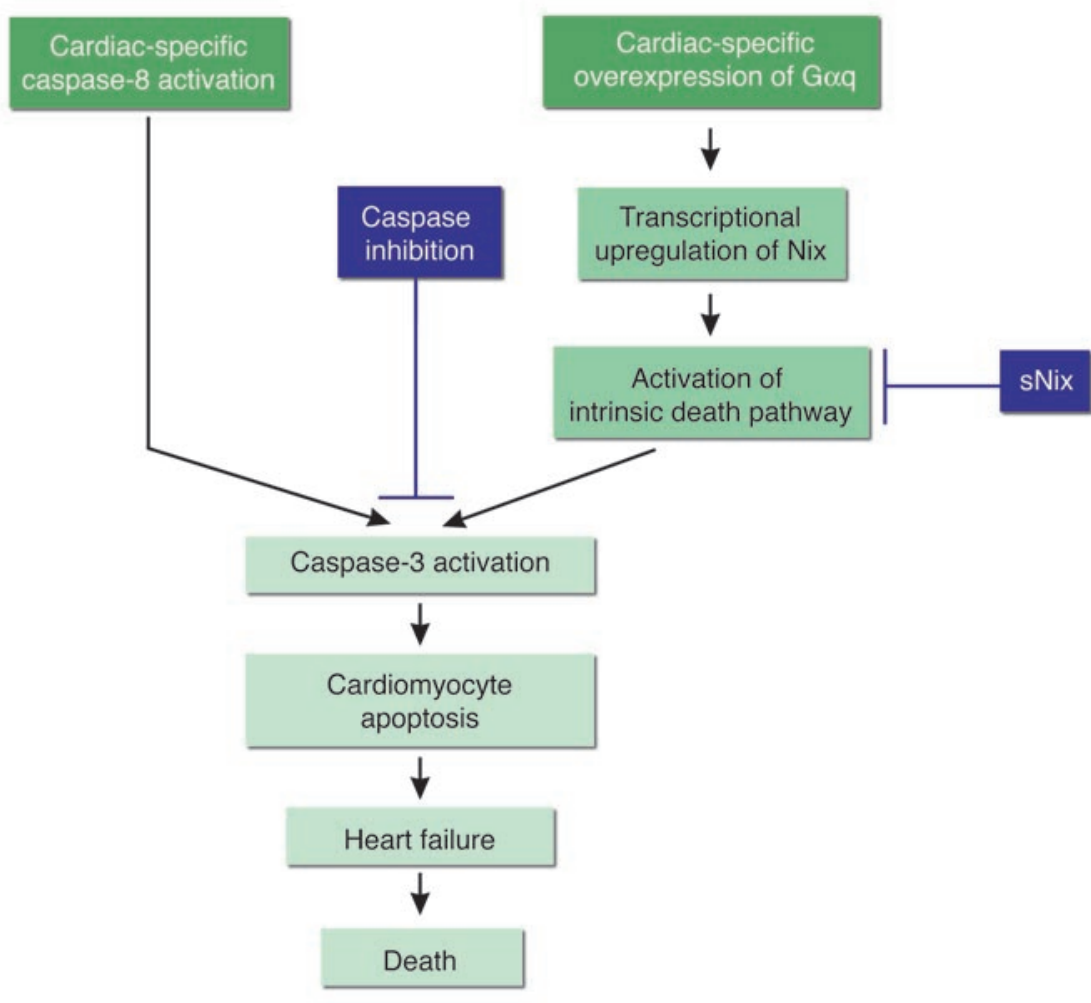

\section{Figure 2}

Cardiomyocyte apoptosis plays a mechanistic role in murine heart failure models. Cardiac-restricted expression of a conditional caspase-8 transgene indicates that low, but abnormal, levels of cardiomyocyte apoptosis $(0.023 \%$ vs. $0.002 \%$ in controls) are sufficient to cause a lethal dilated cardiomyopathy. Similarly, cardiac-specific expression of $\mathrm{G} \alpha \mathrm{q}$ induces the transcriptional activation of the BH3-only-like protein Nix, which results in cardiomyocyte apoptosis and dilated cardiomyopathy. The latter is severely exacerbated and lethal during pregnancy. Caspase inhibitors or expression of sNix, an antiapoptotic Nix splice variant, ameliorate cardiomyopathy in these models. In addition, caspase inhibition and sNix decrease mortality in the Gaq peripartum cardiomyopathy model.

clines (97), although the relative importance of these stimuli in the most common instances of heart failure has not yet been established. Likewise, with the exception of the angiotensin II $\rightarrow$ $\mathrm{G} \alpha \mathrm{q} \rightarrow$ Nix and gp130 $\rightarrow$ STAT $\rightarrow$ Bcl- $x_{\mathrm{L}}$ pathways noted above, little is known about the connections between the myriad of upstream signaling pathways and the central death machinery. Moreover, the pathophysiological significance of some upstream events is poorly understood. For example, strong evidence implicates telo-

apoptosis, which is mediated by the transcriptional induction of Nix/BNip3L, a BH3-only-like protein (82). In addition to this baseline cardiomyopathy, $30 \%$ of pregnant Gaq-transgenic mice exhibit fulminant lethal heart failure accompanied by further increases in apoptosis. Inhibition of apoptosis, either through expression of sNix, an antiapoptotic Nix splice variant (82), or administration of a broad-spectrum caspase inhibitor (83), markedly decreases the severity and mortality of the Gaq peripartum cardiomyopathy.

Hemodynamic overload is a unifying stimulus in multiple forms of heart failure. Several studies provide evidence that inhibition of cardiomyocyte apoptosis lessens the severity of cardiomyopathy resulting from hemodynamic overload. Overexpression of Bcl-2 (84) or ARC (85) inhibits cardiomyopathy induced by prior myocardial infarction. Conversely, loss of a basal survival mechanism can exacerbate cardiomyopathy induced by hemodynamic overload. This is illustrated by a study on mice with ventricularrestricted deletion of gp130 (86), a component of the receptor for several prosurvival cytokines that induces the STAT-dependent transcription of $\mathrm{Bcl}-\mathrm{x}_{\mathrm{L}}$ (87). While these mice are normal at baseline, they develop lethal heart failure following aortic constriction. Taken together, these and other studies $(88,89)$ demonstrate a mechanistic role for cardiomyocyte apoptosis in heart failure.

\section{What pathways mediate cardiomyocyte apoptosis in the failing heart?}

Although the fundamental blueprint for cell death signaling has been highly conserved over more than a billion years of evolution, the precise molecular events - especially upstream ones - are often specific to cell type and/or death stimulus. Relevant stimuli in heart failure probably include stretch (90), ROS (91), $\beta_{1}$-adrenergic agonists $(92,93)$, angiotensin II (94), proinflammatory cytokines (95), cytoskeletal abnormalities (96), and drugs such as anthracy- mere dysfunction as a cause for myocyte apoptosis in heart failure (98-100). However, it remains unclear whether telomere uncapping represents an abortive attempt by cardiomyocytes to reenter the cell cycle or a response to the activation of multiple stress pathways.

Even the importance of each of the central death pathways in heart failure needs to be better defined. For example, while both intrinsic and extrinsic death pathways play significant roles in ischemia-reperfusion injury, little is known about the importance of the extrinsic pathway in heart failure. Similarly, although the ER pathway is known to be activated in failing human hearts (101), the extent to which it plays a mechanistic role in cardiomyocyte demise remains to be determined. The study of mice harboring mutations in various components of the central death machinery, which has been so informative with respect to understanding ischemia-reperfusion injury, should be useful in addressing some of these questions.

\section{Potential therapeutic targets and future questions}

We have described experimental evidence showing that cardiomyocyte apoptosis is a causal component of ischemia-reperfusion injury and heart failure in rodent models. If this paradigm extends to humans, it follows that prevention or inhibition of myocyte death may provide a novel therapeutic approach to these most common and lethal heart syndromes. How might inhibition of cardiomyocyte apoptosis be achieved in a clinical setting?

We will limit our comments to small molecule therapies, which, at present, are the most practical options. In general, receptors and enzymes constitute the molecules most amenable to pharmacological manipulation. Several receptor/enzyme pathways influence survival in cardiomyocytes. In fact, $\beta$-adrenergic receptor antagonists and inhibitors of the angiotensin II axis (angiotensin-converting enzyme inhibitors and angiotensin II type 1 receptor antagonists) are already mainstays in the treatment of heart failure. The 
extent to which the salutary effects of these agents are attributable to their inhibition of apoptosis is not known. Although $\beta_{1}$-selective adrenergic receptor blockers are used primarily to avoid extracardiac side effects, this approach is also consistent with observations that $\beta_{1}$-adrenergic receptors activate death pathways in cardiomyocytes, which are opposed by $\beta_{2}$-adrenergic receptors $(102,103)$.

The serine-threonine kinase Akt is a central molecule in several receptor-mediated survival pathways in cardiomyocytes. Akt inhibits apoptotic signaling at multiple levels including phosphorylation of the BH3-only protein $\mathrm{Bad}(104,105), \operatorname{IKK} \beta$ (a proximal kinase that activates NF- $\mathrm{KB}$ signaling) (106), the proapoptotic transcription factor Foxo3a (107), and perhaps procaspase-9 $(108,109)$. Akt can be activated in cardiomyocytes by receptor tyrosine kinase ligands, such as IGF-1 $(110,111)$, and by exogenously administered thymosin $\beta 4$ (112). IGF- 1 and Akt have been demonstrated to reduce cardiomyocyte apoptosis and infarct size following ischemia-reperfusion and prolonged ischemia $(111,113-117)$. In addition, cardiac-restricted expression of an IGF-1 transgene has beneficial effects on cardiac remodeling following myocardial infarction (114) and in a genetic model of cardiomyopathy (118), although interpretation of these studies is complicated by the fact that myocardial IGF-1 expression was present in the mice beginning in the fetus, which resulted in baseline increases in the number of cardiomyocytes (119). Interestingly, growth hormone, which increases serum IGF-1 levels, was shown in a small clinical study to improve clinical and functional parameters in patients with idiopathic dilated cardiomyopathy (120). Although questions remain concerning the deleterious effects of Akt on the heart (121), the data discussed here raise the possibility that IGF-1 may ameliorate heart failure through inhibition of cardiomyocyte apoptosis. Similarly, activation of the gp130 $\rightarrow$ STAT $\rightarrow$ Bcl- $x_{\mathrm{L}}$ survival pathway with ligands such as cardiotrophin-1 (CT-1) may provide an alternative means of achieving this end (122).

Although manipulation of receptor-mediated upstream pathways is attractive because of their accessibility, this approach can be confounded by redundancy and complicated by undesirable pleiotropic effects. These issues provide a strong rationale for interventions that focus directly on the central death pathways. Where in the central death pathways would inhibition of cardiomyocyte apoptosis be most efficiently achieved? Both central death pathways converge on caspases. Broad-spectrum caspase inhibitors reduce infarct size by $21-52 \%$ and decrease cardiac dysfunction following ischemiareperfusion (123-125, S1). Caspase inhibitors have also been shown to ameliorate cardiac dysfunction and/or inhibit mortality in the caspase- 8 and Gaq models of dilated cardiomyopathy discussed above $(77,83)$. These effects of caspase inhibition correlate with inhibition of cardiomyocyte death, which suggests that the benefit of these agents results from their antiapoptotic properties. Recently, however, caspases have been demonstrated to cleave cardiac contractile proteins and at least 1 cardiac transcription factor (S2-S4). This raises the possibility that caspase inhibitors may also preserve cardiac function independently of their effects on cell death.

The efficacy of caspase inhibitors in these settings poses important questions. Caspase activation in cardiomyocytes occurs after mitochondrial damage and the release of apoptogens. Moreover, as in many systems, cytochrome $c$ release in the myocardium is unaffected by caspase inhibition (S5). Given these mitochondrial abnormalities, it is curious that caspase inhibitors exert such marked improvement on disease pathogenesis. Whether their beneficial effects result from inhibiting upstream caspases, the release of other apoptogens (such as apoptosis-inducing factor and endonuclease $\mathrm{G}$, whose translocation appears to be caspase dependent; refs. S6, S7), or other yet-to-bedescribed mechanisms remains to be determined.

Another example of a small molecule that antagonizes the central death machinery is UCF-1, an inhibitor of the serine protease activity of Omi/HtrA2 (S8). Unexpectedly, UCF-1 markedly inhibits cardiomyocyte apoptosis and reduces infarct size following ischemia-reperfusion by maintaining XIAP levels (S9). These data indicate that relief of inhibitory pathways is critical for apoptosis to proceed in cardiomyocytes and suggest that strategies built around maintaining inhibition may be cardioprotective.

Despite the efficacy of postmitochondrial inhibition of apoptosis in these examples, an even more effective strategy may be to intercept apoptotic signaling upstream of the mitochondria. While a premitochondrial approach might be limited by redundancy, it offers the important advantage of preventing mitochondrial dysfunction. In fact, cells lacking both Bax and Bak, either one of which is required for upstream death signals to gain access to the mitochondria, exhibit long-term protection against multiple noxious stimuli (S10, S11). Premitochondrial inhibition may require drugs that interfere with interactions between $\mathrm{BH} 3$-only (e.g., Bid) and multidomain proapoptotic $\mathrm{Bcl}-2$ proteins (e.g., Bax and Bak) (Figure 1). Another attractive possibility is provided by the endogenous inhibitor ARC. ARC would provide premitochondrial inhibition of both extrinsic and intrinsic pathways and preserve mitochondrial function (51, S12). In addition, the relatively restricted expression pattern of endogenous ARC (52) may offer a means to avoid potential carcinogenic effects of diffuse long-term inhibition of apoptosis. A major outstanding issue is how best to exploit endogenous ARC as a therapeutic agent.

\section{Conclusions}

Our understanding of the significance of myocyte loss during heart failure has increased substantially since this phenomenon was initially observed by cardiac pathologists. Molecular and genetic studies demonstrate clearly that cardiomyocyte apoptosis is a critical process in the pathogenesis of heart failure in rodent models. If this paradigm extends to humans, apoptosis will be a logical target for novel therapies. Despite success in establishing a mechanistic link between apoptosis and heart failure, our knowledge of the precise molecular mechanisms that regulate cell death specifically in this syndrome remains rudimentary. An understanding of these mechanisms will be indispensable for the rational design of future antiapoptotic therapies.

\section{Acknowledgments}

We are indebted to Thierry H. LeJemtel and James Scheuer for critical reading of the manuscript. This work was supported by NIH R01 grants HL60665, HL61550, and HL73732 (to R.N. Kitsis) and a Wellcome Trust Advanced Fellowship (to R.S.-Y. Foo). R.N. Kitsis was also supported by The Dr. Gerald and Myra Dorros Chair in Cardiovascular Disease of the Albert Einstein College of Medicine and the Monique Weill-Caulier Career Scientist Award.

Due to space constraints, a number of important references could not be included in this article. References S1-S12 are available online with this article; doi:10.1172/JCI200524569DS1.

Address correspondence to Richard N. Kitsis, Albert Einstein College of Medicine, 1300 Morris Park Avenue, Bronx, New York 10461, USA. Phone: (718) 430-2609; Fax: (718) 430-8989; E-mail: kitsis@aecom.yu.edu. 
1. Mann, D.L. 1999. Mechanisms and models in heart failure: a combinatorial approach. Circulation. 100:999-1008.

2. Lower, R. 1669. Tractatus de corde. Item de motu et colore sanguinis et chyli in eum transitu. Jo. Redmayne for Jacob Allestry. London, United Kingdom.

3. Lefkowitz, R.J., Rockman, H.A., and Koch, W.J. 2000 Catecholamines, cardiac beta-adrenergic receptors, and heart failure. Circulation. 101:1634-1637.

4. Marks, A.R. 2002. Ryanodine receptors, FKBP12, and heart failure. Front. Biosci. 7:d970-d977.

5. Luo, W., et al. 1994. Targeted ablation of the phospholamban gene is associated with markedly enhanced myocardial contractility and loss of betaagonist stimulation. Circ. Res. 75:401-409.

6. Chien, K.R. 1999. Stress pathways and heart failure. Cell. 98:555-558.

7. Nakao, K., Minobe, W., Roden, R., Bristow, M.R. and Leinwand, L.A. 1997. Myosin heavy chain gene expression in human heart failure. J. Clin. Invest. 100:2362-2370.

8. Taegtmeyer, H. 2002. Switching metabolic genes to build a better heart. Circulation. 106:2043-2045.

9. Kelly, D.P., and Strauss, A.W. 1994. Inherited cardiomyopathies. N. Engl. J. Med. 330:913-919.

10. Danial, N.N., and Korsmeyer, S.J. 2004. Cell death: critical control points. Cell. 116:205-219.

11. Yuan, J., Lipinski, M., and Degterev, A. 2003. Diversity in the mechanisms of neuronal cell death. Neuron. 40:401-413.

12. Zong, W.X., Ditsworth, D., Bauer, D.E., Wang, Z.Q., and Thompson, C.B. 2004. Alkylating DNA damage stimulates a regulated form of necrotic cell death. Genes Dev. 18:1272-1282.

13. Klionsky, D.J., and Emr, S.D. 2000. Autophagy as a regulated pathway of cellular degradation. Science. 290:1717-1721.

14. Shintani, T., and Klionsky, D.J. 2004. Autophagy in health and disease: a double-edged sword. Science. 306:990-995

15. Ashkenazi, A., and Dixit, V.M. 1998. Death receptors: signaling and modulation. Science. 281:1305-1308.

16. Kischkel, F.C., et al. 1995. Cytotoxicity-dependent APO-1 (Fas/CD95)-associated proteins form death-inducing signaling complex (DISC) with the receptor. EMBO J. 14:5579-5588.

17. Boldin, M.P., Goncharov, T.M., Goltsev, Y.V., and Wallach, D. 1996. Involvement of MACH, a nove MORT1/FADD-interacting protease, in Fas/ APO-1- and TNF receptor-induced cell death. Cell. 85:803-815.

18. Muzio, M., et al. 1996. FLICE, a novel FADDhomologous ICE/CED-3-like protease, is recruited to the CD95 (Fas/APO-1) death-inducing signaling complex. Cell. 85:817-827.

19. Boatright, K.M., et al. 2003. A unified model for apical caspase activation. Mol. Cell. 11:529-541.

20. Crow, M.T., Mani, K., Nam, Y.J., and Kitsis, R.N 2004. The mitochondrial death pathway and cardiac myocyte apoptosis. Circ. Res. 95:957-970.

21. Liu, X., Kim, C.N., Yang, J., Jemmerson, R., and Wang, X. 1996. Induction of apoptotic program in cell-free extracts: requirement for dATP and cytochrome c. Cell. 86:147-157.

22. Kluck, R.M., Bossy-Wetzel, E., Green, D.R., and Newmeyer, D.D. 1997. The release of cytochrome c from mitochondria: a primary site for $\mathrm{Bcl}-2$ regulation of apoptosis. Science. 275:1132-1136.

23. Du, C., Fang, M., Li, Y., Li, L., and Wang, X. 2000. Smac, a mitochondrial protein that promotes cytochrome c-dependent caspase activation by eliminating IAP inhibition. Cell. 102:33-42.

24. Verhagen, A.M., et al. 2000. Identification of DIAB$\mathrm{LO}$, a mammalian protein that promotes apoptosis by binding to and antagonizing IAP proteins. Cell. 102:43-53.

25. Suzuki, Y., et al. 2001. A serine protease, HtrA2, is released from the mitochondria and interacts with
XIAP, inducing cell death. Mol. Cell. 8:613-621.

26. Susin, S.A., et al. 1999. Molecular characterization of mitochondrial apoptosis-inducing factor. Nature. 397:441-446.

27. Li, L.Y., Luo, X., and Wang, X. 2001. Endonuclease $\mathrm{G}$ is an apoptotic DNase when released from mitochondria. Nature. 412:95-99.

28. Zou, H., Henzel, W.J., Liu, X., Lutschg, A., and Wang, X. 1997. Apaf-1, a human protein homologous to C. elegans CED-4, participates in cytochrome c-dependent activation of caspase-3. Cell. 90:405-413.

29. Li, P., et al. 1997. Cytochrome c and dATP-dependent formation of Apaf-1/caspase- 9 complex initiates an apoptotic protease cascade. Cell. 91:479-489.

30. Srinivasula, S.M., Ahmad, M., Fernandes-Alnemri, T., and Alnemri, E.S. 1998. Autoactivation of procaspase- 9 by Apaf-1-mediated oligomerization. Mol. Cell. 1:949-957.

31. Hu, Y., Ding, L., Spencer, D.M., and Nunez, G 1998. WD-40 repeat region regulates Apaf-1 selfassociation and procaspase-9 activation. J. Biol. Chem. 273:33489-33494.

32. Saleh, A., Srinivasula, S.M., Acharya, S., Fishel, R., and Alnemri, E.S. 1999. Cytochrome c and dATPmediated oligomerization of Apaf-1 is a prerequisite for procaspase- 9 activation. J. Biol. Chem. 274:17941-17945.

33. Zou, H., Li, Y., Liu, X., and Wang, X. 1999. An APAF1.cytochrome c multimeric complex is a functional apoptosome that activates procaspase- 9 . J. Biol. Chem. 274:11549-11556.

34. Qin, H., et al. 1999. Structural basis of procaspase-9 recruitment by the apoptotic protease-activating factor 1. Nature. 399:549-557.

35. Vaughn, D.E., Rodriguez, J., Lazebnik, Y., and Joshua-Tor, L. 1999. Crystal structure of Apaf-1 caspase recruitment domain: an alpha-helical Greek key fold for apoptotic signaling. J. Mol. Biol. 293:439-447.

36. Zhou, P., Chou, J., Olea, R.S., Yuan, J., and Wagner, G. 1999. Solution structure of Apaf-1 CARD and its interaction with caspase-9 CARD: a structural basis for specific adaptor/caspase interaction. Proc. Natl. Acad. Sci. U. S. A. 96:11265-11270.

37. Day, C.L., Dupont, C., Lackmann, M., Vaux, D.L., and Hinds, M.G. 1999. Solution structure and mutagenesis of the caspase recruitment domain (CARD) from Apaf-1. Cell Death Differ. 6:1125-1132.

38. Jiang, X., and Wang, X. 2000. Cytochrome c promotes caspase- 9 activation by inducing nucleotide binding to Apaf-1. J. Biol. Chem. 275:31199-31203.

39. Acehan, D., et al. 2002. Three-dimensional structure of the apoptosome: implications for assembly, procaspase- 9 binding, and activation. Mol. Cell. 9:423-432.

40. Luo, X., Budihardjo, I., Zou, H., Slaughter, C., and Wang, X. 1998. Bid, a Bcl2 interacting protein, mediates cytochrome $\mathrm{c}$ release from mitochondria in response to activation of cell surface death receptors. Cell. 94:481-490.

41. Li, H., Zhu, H., Xu, C.J., and Yuan, J. 1998. Cleavage of BID by caspase 8 mediates the mitochondrial damage in the Fas pathway of apoptosis. Cell. 94:491-501.

42. Gross, A., et al. 1999. Caspase cleaved BID targets mitochondria and is required for cytochrome $c$ release, while BCL-XL prevents this release but not tumor necrosis factor-R1/Fas death. J. Biol. Chem. 274:1156-1163.

43. Peter, M.E. 2004. The flip side of FLIP. Biochem. J. 382:e1-e3.

44. Sawada, M., et al. 2003. Ku70 suppresses the apoptotic translocation of Bax to mitochondria. Nat Cell Biol. 5:320-329.

45. Guo, B., et al. 2003. Humanin peptide suppresses apoptosis by interfering with Bax activation. Nature, 423:456-461.

46. Sun, C., et al. 2000. NMR structure and mutagenesis of the third Bir domain of the inhibitor of apoptosis protein XIAP. J. Biol. Chem. 275:33777-33781.

47. Chai, J., et al. 2001. Structural basis of caspase-7 inhibition by XIAP. Cell. 104:769-780.

48. Huang, Y., et al. 2001. Structural basis of caspase inhibition by XIAP: differential roles of the linker versus the BIR domain. Cell. 104:781-790.

49. Riedl, S.J., et al. 2001. Structural basis for the inhibition of caspase- 3 by XIAP. Cell. 104:791-800.

50. Shiozaki, E.N., et al. 2003. Mechanism of XIAP-mediated inhibition of caspase-9. Mol. Cell. 11:519-527.

51. Nam, Y.J., et al. 2004. Inhibition of both the extrinsic and intrinsic death pathways through nonhomotypic death-fold interactions. Mol. Cell. 15:901-912.

52. Koseki, T., Inohara, N., Chen, S., and Nunez, G. 1998. ARC, an inhibitor of apoptosis expressed in skeletal muscle and heart that interacts selectively with caspases. Proc. Natl. Acad. Sci. U. S. A. 95:5156-5160.

53. Gustafsson, A.B., Tsai, J.G., Logue, S.E., Crow, M.T., and Gottlieb, R.A. 2004. Apoptosis repressor with caspase recruitment domain protects against cell death by interfering with Bax activation. J. Biol. Chem. 279:21233-21238.

54. Potts, P.R., Singh, S., Knezek, M., Thompson, C.B., and Deshmukh, M. 2003. Critical function of endogenous XIAP in regulating caspase activation during sympathetic neuronal apoptosis. J. Cell Biol. 163:789-799.

55. Yang, Q.H., Church-Hajduk, R., Ren, J., Newton, M.L., and Du, C. 2003. Omi/HtrA2 catalytic cleavage of inhibitor of apoptosis (IAP) irreversibly inactivates IAPs and facilitates caspase activity in apoptosis. Genes Dev. 17:1487-1496.

56. Scorrano, L., et al. 2003. BAX and BAK regulation of endoplasmic reticulum Ca2+: a control point for apoptosis. Science. 300:135-139.

57. Morishima, N., Nakanishi, K., Tsuchiya, K., Shibata, T., and Seiwa, E. 2004. Translocation of Bim to the endoplasmic reticulum (ER) mediates ER stress signaling for activation of caspase-12 during ER stressinduced apoptosis. J. Biol. Chem. 279:50375-50381.

58. Oakes, S.A., et al. 2005. Proapoptotic BAX and BAK regulate the type 1 inositol trisphosphate receptor and calcium leak from the endoplasmic reticulum. Proc. Natl. Acad. Sci. U. S. A. 102:105-110.

59. Halestrap, A.P., McStay, G.P., and Clarke, S.J. 2002. The permeability transition pore complex: another view. Biochimie. 84:153-166.

60. Scorrano, L., et al. 2002. A distinct pathway remodels mitochondrial cristae and mobilizes cytochrome c during apoptosis. Dev. Cell. 2:55-67.

61. Nakagawa, T., et al. 2000. Caspase-12 mediates endoplasmic-reticulum-specific apoptosis and cytotoxicity by amyloid-beta. Nature. 403:98-103.

62. Szegezdi, E., Fitzgerald, U., and Samali, A. 2003. Caspase-12 and ER-stress-mediated apoptosis: the story so far. Ann. N. Y. Acad. Sci. 1010:186-194.

63. Nakagawa, T., and Yuan, J. 2000. Cross-talk between two cysteine protease families. Activation of caspase-12 by calpain in apoptosis. J. Cell Biol. 150:887-894.

64. Morishima, N., Nakanishi, K., Takenouchi, H., Shibata, T., and Yasuhiko, Y. 2002. An endoplasmic reticulum stress-specific caspase cascade in apoptosis. Cytochrome c-independent activation of caspase-9 by caspase-12. J. Biol. Chem. 277:34287-34294

65. Saleh, M., et al. 2004. Differential modulation of endotoxin responsiveness by human caspase- 12 polymorphisms. Nature. 429:75-79.

66. Gottlieb, R.A., Burleson, K.O., Kloner, R.A., Babior, B.M., and Engler, R.L. 1994. Reperfusion injury induces apoptosis in rabbit cardiomyocytes. J. Clin. Invest. 94:1621-1628.

67. Fliss, H., and Gattinger, D. 1996. Apoptosis in ischemic and reperfused rat myocardium. Circ. Res. 79:949-956.

68. Lee, P., et al. 2003. Fas pathway is a critical media- 
tor of cardiac myocyte death and MI during ischemia-reperfusion in vivo. Am. J. Physiol. Heart Circ. Physiol. 284:H456-H463.

69. Jeremias, I., et al. 2000. Involvement of CD95/ Apo1/Fas in cell death after myocardial ischemia. Circulation. 102:915-920.

70. Peng, C.-F., et al. 2001. Multiple independent mutations in apoptotic signaling pathways markedly decrease infarct size due to myocardial ischemia-reperfusion [abstract]. Circulation. 104(Suppl. II):II-187.

71. Brocheriou, V., et al. 2000. Cardiac functional improvement by a human $\mathrm{Bcl}-2$ transgene in a mouse model of ischemia/reperfusion injury. J. Gene Med. 2:326-333.

72. Chen, Z., Chua, C.C., Ho, Y.S., Hamdy, R.C., and Chua, B.H. 2001. Overexpression of Bcl-2 attenuates apoptosis and protects against myocardial I/R injury in transgenic mice. Am. J. Physiol. Heart Circ. Physiol. 280:H2313-H2320.

73. Guerra, S., et al. 1999. Myocyte death in the failing human heart is gender dependent. Circ. Res. 85:856-866.

74. Hein, S., et al. 2003. Progression from compensated hypertrophy to failure in the pressure-overloaded human heart: structural deterioration and compensatory mechanisms. Circulation. 107:984-991.

75. Olivetti, G., et al. 1997. Apoptosis in the failing human heart. N. Engl. J. Med. 336:1131-1141.

76. Saraste, A., et al. 1999. Cardiomyocyte apoptosis and progression of heart failure to transplantation. Eur. J. Clin. Invest. 29:380-386.

77. Wencker, D., et al. 2003. A mechanistic role for cardiac myocyte apoptosis in heart failure. Cell. 111:1497-1504.

78. Dorn, G.W., 2nd, and Brown, J.H. 1999. Gq signaling in cardiac adaptation and maladaptation. Trends Cardiovasc. Med. 9:26-34.

79. D’Angelo, D.D., et al. 1997. Transgenic Galphaq overexpression induces cardiac contractile failure in mice. Proc. Natl. Acad. Sci. U. S. A. 94:8121-8126.

80. Adams, J.W., et al. 1998. Enhanced Galphaq signaling: a common pathway mediates cardiac hypertrophy and apoptotic heart failure. Proc. Natl. Acad. Sci. U. S. A. 95:10140-10145.

81. Sakata, Y., Hoit, B.D., Liggett, S.B., Walsh, R.A., and Dorn, G.W., 2nd. 1998. Decompensation of pressure-overload hypertrophy in $\mathrm{G}$ alpha q-overexpressing mice. Circulation. 97:1488-1495.

82. Yussman, M.G., et al. 2002. Mitochondrial death protein Nix is induced in cardiac hypertrophy and triggers apoptotic cardiomyopathy. Nat. Med. 8:725-730.

83. Hayakawa, Y., et al. 2003. Inhibition of cardiac myocyte apoptosis improves cardiac function and abolishes mortality in the peripartum cardiomyopathy of Galpha(q) transgenic mice. Circulation. 108:3036-3041.

84. Chatterjee, S., et al. 2002. Viral gene transfer of the antiapoptotic factor $\mathrm{Bcl}-2$ protects against chronic postischemic heart failure. Circulation. 106:I212-I217.

85. Chatterjee, S., et al. 2003. Blocking the development of postischemic cardiomyopathy with viral gene transfer of the apoptosis repressor with caspase recruitment domain. J. Thorac. Cardiovasc. Surg. 125:1461-1469.

86. Hirota, H., et al. 1999. Loss of a gp130 cardiac muscle cell survival pathway is a critical event in the onset of heart failure during biomechanical stress. Cell. 97:189-198.

87. Fujio, Y., Kunisada, K., Hirota, H., Yamauchi-Takihara, K., and Kishimoto, T. 1997. Signals through gp130 upregulate bcl-x gene expression via STAT1- binding cis-element in cardiac myocytes. Cell. 99:2898-2905.

88. Xing, H., Zhang, S., Weinheimer, C., Kovacs, A., and Muslin, A.J. 2000. 14-3-3 proteins block apoptosis and differentially regulate MAPK cascades. EMBOJ. 19:349-358.

89. Zhang, D., et al. 2000. TAK1 is activated in the myocardium after pressure overload and is sufficient to provoke heart failure in transgenic mice. Nat. Med. 6:556-563.

90. Cheng, W., et al. 1995. Stretch-induced programmed myocyte cell death. Cell. 96:2247-2259.

91. Ide, T., et al. 2000. Direct evidence for increased hydroxyl radicals originating from superoxide in the failing myocardium. Circ. Res. 86:152-157.

92. Communal, C., Singh, K., Pimentel, D.R., and Colucci, W.S. 1998. Norepinephrine stimulates apoptosis in adult rat ventricular myocytes by activation of the beta-adrenergic pathway. Circulation. 98:1329-1334.

93. Shizukuda, Y., et al. 1998. beta-adrenergic stimulation causes cardiocyte apoptosis: influence of tachycardia and hypertrophy. Am. J. Physiol. 275:H961-H968.

94. Cigola, E., Kajstura, J., Li, B., Meggs, L.G., and Anversa, P. 1997. Angiotensin II activates programmed myocyte cell death in vitro. Exp. Cell Res. 231:363-371.

95. Mann, D.L. 1999. Inflammatory mediators in heart failure: homogeneity through heterogeneity. Lancet. 353:1812-1813.

96. Nikolova, V., et al. 2004. Defects in nuclear structure and function promote dilated cardiomyopathy in lamin A/C-deficient mice. Cell. 113:357-369.

97. Wang, L., Ma, W., Markovich, R., Chen, J.W., and Wang, P.H. 1998. Regulation of cardiomyocyte apoptotic signaling by insulin-like growth factor I. Circ. Res. 83:516-522.

98. Oh, H., et al. 2001. Telomerase reverse transcriptase promotes cardiac muscle cell proliferation, hypertrophy, and survival. Proc. Natl. Acad. Sci. U. S. A. 98:10308-10313

99. Leri, A., et al. 2003. Ablation of telomerase and telomere loss leads to cardiac dilatation and heart failure associated with $\mathrm{p} 53$ upregulation. $E M B O J$. 22:131-139.

100.Oh, H., et al. 2003. Telomere attrition and Chk2 activation in human heart failure. Proc. Natl. Acad. Sci. U. S. A. 100:5378-5383.

101. Okada, K., et al. 2004. Prolonged endoplasmic reticulum stress in hypertrophic and failing heart after aortic constriction: possible contribution of endoplasmic reticulum stress to cardiac myocyte apoptosis. Circulation. 110:705-712.

102. Chesley, A., et al. 2000. The beta(2)-adrenergic receptor delivers an antiapoptotic signal to cardiac myocytes through $\mathrm{G}(\mathrm{i})$-dependent coupling to phosphatidylinositol 3'-kinase. Circ. Res. 87:1172-1179.

103.Xiao, R.P. 2001. Beta-adrenergic signaling in the heart: dual coupling of the beta2- adrenergic receptor to $\mathrm{G}(\mathrm{s})$ and $\mathrm{G}(\mathrm{i})$ proteins. Sci. STKE. 2001:RE15.

104.Datta, S.R., et al. 1997. Akt phosphorylation of BAD couples survival signals to the cell-intrinsic death machinery. Cell. 91:231-241.

105.Mehrhof, F.B., et al. 2001. In cardiomyocyte hypoxia, insulin-like growth factor-I-induced antiapoptotic signaling requires phosphatidylinositol3-OH-kinase-dependent and mitogen-activated protein kinase-dependent activation of the transcription factor cAMP response element-binding protein. Circulation. 104:2088-2094.

106. Romashkova, J.A., and Makarov, S.S. 1999. NFkappaB is a target of AKT in anti-apoptotic PDGF signalling. Nature. 401:86-90
107. Brunet, A., et al. 1999. Akt promotes cell survival by phosphorylating and inhibiting a Forkhead transcription factor. Cell. 96:857-868.

108. Cardone, M.H., et al. 1998. Regulation of cell death protease caspase-9 by phosphorylation. Science. 282:1318-1321.

109.Fujita, E., et al. 1999. Akt phosphorylation site found in human caspase- 9 is absent in mouse caspase-9. Biochem. Biophys. Res. Commun. 264:550-555.

110. Matsui, T., et al. 1999. Adenoviral gene transfer of activated phosphatidylinositol 3'-kinase and Akt inhibits apoptosis of hypoxic cardiomyocytes in vitro. Circulation. 100:2373-2379.

111.Fujio, Y., Nguyen, T., Wencker, D., Kitsis, R.N., and Walsh, K. 2000. Akt promotes survival of cardiomyocytes in vitro and protects against ischemiareperfusion injury in mouse heart. Circulation. 101:660-667.

112.Bock-Marquette, I., Saxena, A., White, M.D., Dimaio, J.M., and Srivastava, D. 2004. Thymosin beta4 activates integrin-linked kinase and promotes cardiac cell migration, survival and cardiac repair. Nature. 432:466-472.

113. Buerke, M., et al. 1995. Cardioprotective effect of insulin-like growth factor I in myocardial ischemia followed by reperfusion. Proc. Natl. Acad. Sci. U. S. A. 92:8031-8035.

114.Li, Q., et al. 1997. Overexpression of insulin-like growth factor- 1 in mice protects from myocyte death after infarction, attenuating ventricular dilation, wall stress, and cardiac hypertrophy. Cell. 100:1991-1999.

115.Miao, W., Luo, Z., Kitsis, R.N., and Walsh, K. 2000. Intracoronary, adenovirus-mediated Akt gene transfer in heart limits infarct size following ischemia-reperfusion injury in vivo. J. Mol. Cell. Cardiol. 32:2397-2402.

116. Matsui, T., et al. 2001. Akt activation preserves cardiac function and prevents injury after transient cardiac ischemia in vivo. Circulation. 104:330-335.

117. Chao, W., et al. 2003. Strategic advantages of insulin-like growth factor-I expression for cardioprotection. J. Gene Med. 5:277-286.

118. Welch, S., et al. 2002. Cardiac-specific IGF-1 expression attenuates dilated cardiomyopathy in tropomodulin-overexpressing transgenic mice. Circ. Res. 90:641-648.

119. Reiss, K., et al. 1996. Overexpression of insulinlike growth factor- 1 in the heart is coupled with myocyte proliferation in transgenic mice. Proc. Natl. Acad. Sci.U. S. A. 93:8630-8635.

120.Fazio, S., et al. 1996. A preliminary study of growth hormone in the treatment of dilated cardiomyopathy. N. Engl. J. Med. 334:809-814

121. Matsui, T., et al. 2002. Phenotypic spectrum caused by transgenic overexpression of activated Akt in the heart. J. Biol. Chem. 277:22896-22901.

122.Pennica, D., Wood, W.I., and Chien, K.R. 1996. Cardiotrophin-1: a multifunctional cytokine that signals via LIF receptor-gp 130 dependent pathways. Cytokine Growth Factor Rev. 7:81-91.

123.Yaoita, H., Ogawa, K., Maehara, K., and Maruyama, Y. 1998. Attenuation of ischemia/reperfusion injury in rats by a caspase inhibitor. Circulation. 97:276-281.

124. Holly, T.A., et al. 1999. Caspase inhibition reduces myocyte cell death induced by myocardial ischemia and reperfusion in vivo. J. Mol. Cell. Cardiol. 31:1709-1715.

125.Huang, J.Q., Radinovic, S., Rezaiefar, P., and Black, S.C. 2000. In vivo myocardial infarct size reduction by a caspase inhibitor administered after the onset of ischemia. Eur. J. Pharmacol. 402:139-142. 